\title{
Path Navigation in ACO Using Mobile Robot
}

\author{
P. Hema Suganthi ME-CSE, Mrs. K. Subha M. Tech.
}

Surya Group of Institutions, Chennai, Tamil Nadu, India

\begin{abstract}
How to cite this paper: $\mathrm{P}$. Hema Suganthi | Mrs. K. Subha "Path Navigation in ACO Using Mobile Robot" Published in International Journal of Trend in Scientific Research and Development

(ijtsrd), ISSN: 2456-

6470, Volume-3 |

Issue-3, April 2019, pp.164-169, URL: http://www.ijtsrd.co $\mathrm{m} /$ papers/ijtsrd216

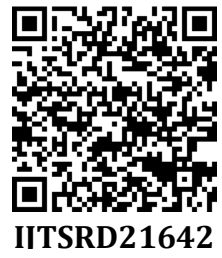
42.pdf

Copyright (C) 2019 by author(s) and International Journal of Trend in Scientific Research and Development Journal. This is an Open Access article distributed under the terms of the Creative Commons

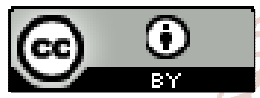
Attribution License (CC BY 4.0) (http://creativecommons.org/licenses/ by/4.0)

\section{INTRODUCTION}

Mobile robot path planning problem is to find the optimal motion path from a given starting point to the end point, which satisfies that robots can be safe and collision-free to bypass all the obstacles in the process of movement According to the degree of robot mastery of the working space environment and the application background, there exists multiple methods for path planning. Including artificial potential field method [1], fuzzy logic method [2], neural network [3] and genetic algorithm [4], etc. The disadvantages of artificial potential field method are local optimum and local optimization and deadlock problem. Fuzzy logic method can overcome the local minimum of the potential field method, but with the increase of the input quantity, the reasoning rules will expand rapidly.

Neural network method has good learning ability, but its network structure is huge. Genetic algorithm has overcome the problem of local minimum, but due to the large amount of storage space generated in the process of evolution, the operation speed is low. [5] Firstly proposed the ant colony algorithm. As the ant colony algorithm has the advantages of positive feedback, parallelism and strong robustness, it has been widely used for path planning in the different complex environments [6-10]. [11] utilized the Global path planning as applied to mobile robotics ant colony algorithm as an approached in a similar fashion as classic optimization problems involving combinational constraints. [12] proposed a method of moving target in complex obstacle environment, and the navigation path planning of robot is carried out based on the improved ant colony algorithm. [13] maximized the leading role of the best individuals of ant colony to improve the convergence speed of path planning algorithm. [14] presented a principle of eliminating the path nodes to improve the speed of the algorithm and simplify the objective function of traditional path planning.

In order to solve the problems of the traditional colony ant algorithm in sensitivity to the initial value and the tendency to fall into local optimum, while maintaining its advantages of fast convergence speed, a method of path planning based on improved ant colony algorithm is proposed in this paper. A hybrid ant colony which is composed of common ants and exploratory ants is adopted to avoid the algorithm being trapped into local optimum. And we improve the pheromone update mechanism to increase the convergence speed. The method is suitable for the path planning in complex terrain environment. The simulation results show that the method is practical and efficient.

\section{ENVIRONMENT MODELLING}

The workspace in this paper is modelled as a grid map, which is represented by a collection of discrete cells, as shown in Fig. 1. Each grid is given an unique label number. The range of activities of ants is a two-dimensional surface enclosed within a specified boundary, where a limited number of static obstacles are distributed. The active area of ants is divided into grids with the same size. A Cartesian coordinate system is established to identify the workspace. The coordinate of the S-th grid is denoted as 
$(x, y)$, where $x=S \% N, y=M-[S / N], M$ is the number of rows in a grid map and $\mathrm{N}$ is the number of columns in a grid map.

\begin{tabular}{|l|l|l|l|l|l|l|l|l|l|}
\hline 10 & 2 & 3 & 4 & 5 & 6 & 7 & 8 & 9 & 10 \\
\hline 11 & 12 & 13 & 14 & 15 & 16 & 17 & 18 & 19 & 20 \\
\hline 21 & 22 & 23 & 24 & 25 & 26 & 27 & 28 & 29 & 30 \\
\hline 31 & 32 & 33 & 34 & 35 & 36 & 37 & 38 & 39 & 40 \\
\hline 41 & 42 & 43 & 44 & 45 & 46 & 47 & 48 & 49 & 50 \\
\hline 51 & 52 & 53 & 54 & 55 & 56 & 57 & 58 & 59 & 60 \\
\hline 61 & 62 & 63 & 64 & 65 & 66 & 67 & 68 & 69 & 70 \\
\hline 71 & 72 & 73 & 74 & 75 & 76 & 77 & 78 & 79 & 80 \\
\hline 81 & 82 & 83 & 84 & 85 & 86 & 87 & 88 & 89 & 90 \\
\hline 91 & 92 & 93 & 94 & 95 & 96 & 97 & 98 & 99 & 100 \\
\hline 0
\end{tabular}

Fig.1 Grid Map

III. ROBOT PATH PLANNING METHOD BASED ON ANT COLONY ALGORITHM

Ant colony algorithm is a kind of bionic algorithm, which simulates the path planning method foraging behaviour of ants. Ants will release pheromones to the environment in the process of searching for food. Ants can perceive the existence of pheromones in a certain range, and move in the direction of high concentration of pheromones. The path of high pheromone concentration can attract more ants, which shows the phenomenon of positive feedback, and finally the whole ant colony can find the optimal path from the nest to the food source.

Artificial ant colony model is utilized to solve the path planning problem in this paper. The main characteristics of artificial ant colony are as follows. At each iteration, ants can only choose the nodes which they have not passed through recently to avoid ants going round in circles. Ants choose the path according to the transfer probability. After each iteration, pheromones of the paths through which ants reach the food source are updated. And pheromone will evaporate over time. Let $m$ be the number of ants in the ant colony. $\tau_{i j}(t)$ is the pheromone concentration of the path from the $i$-th grid to $j$-th grid of time $t$. In the initialization phase of the algorithm, let $\tau_{i j}(0)=C$, where $C$ is a constant. For the $\mathrm{k}$-th ant the probability $p_{i j}^{k}(t)$ of time to move from i-th grid to $\mathrm{j}$-th grid is defined as follows [5].

$p_{i j}^{k}=\left\{\begin{array}{cc}\frac{\tau_{i j}^{\alpha}(t) * \eta_{i j}^{\beta}(t)}{\sum_{s \varepsilon a l l o w e d} \tau_{i s}^{\alpha}(t) * \eta_{i s}^{\beta}(t), \text { if je allowed }} k \quad \text { (1) } \\ 0, \text { otherwise }\end{array}\right\}$

Where allowed $_{k}$ is the set of the remaining feasible states of the k-th ant. $\eta_{i j}(t)$ is the local heuristic function of visibility. $\alpha, \beta$ Represent the affecting weight of $\tau_{i j}(t)$ and $\eta_{i j}(t)$ on the transition probability, respectively. The pheromone update formula is defined as follows [5].

$$
\begin{gathered}
\tau_{i j}(t+n)=(1-\rho) * \tau_{i j}(t)+\Delta \tau_{i j}(t) \\
\Delta \tau_{i j}(t)-\sum_{k=1}^{m} \Delta \tau_{i j}(t)
\end{gathered}
$$

Where $\rho(\rho \epsilon(0,1))$ is the pheromone evaporation coefficient. in $\Delta \tau_{t j}(t)$ is pheromone increment of the path $<\mathrm{i}, \mathrm{j}>$ in the current iteration. $\Delta \tau_{i j}^{k}(t)$ is pheromone increment which the $k$-th ant release on the path $<i, j>$.

$$
\Delta \tau_{i j}(t)=\left\{\begin{array}{c}
\frac{Q}{L_{k}}, i_{i} j \in L_{k} \\
0, \text { otherwise }
\end{array}\right\}
$$

Where $Q$ is a constant, which represents the amount of the pheromone increment. $L_{k}$ is the length of the path that the $\mathrm{k}$-the ant has passed in the current iteration.

\section{IMPROVED PATH PLANNING METHOD BASED ON ANT COLONY ALGORITHM}

A. Improved Ant Colony Model

By the path planning method based on ant colony algorithm, ants select the path according to pheromone concentration on it. The pheromone concentration on a path is associated with the number of the ants passing through the path, which makes the algorithm easy to trap into local optimum. In order to avoid this drawback, a hybrid ant colony which is composed of common ants and exploratory ants is adopted in this paper. Meanwhile, in order to improve the convergence speed of the ant colony algorithm, pheromone update mechanism is improved in this paper. At each iteration, the ants which have found the shortest path in the iteration are labelled as elite ants. The path selection mechanism of ant colony algorithm is implemented by common ants. The path selection mechanism of exploratory ants is defined as follows. For the k-th exploratory ant the probability $p_{i j}^{k}(t)$ of time $t$ to move from i-th grid to $\mathrm{j}$-th grid is defined as follows.

$p_{i j}^{k}(t)=\left\{\begin{array}{c}\frac{\eta_{i j}^{\beta}(t) / \tau_{i j}^{\alpha}(t)}{\sum_{s \varepsilon a l l o w e d} \eta_{i s}^{\beta}(t) / \tau_{i s}^{\alpha}(t)}, j \in \text { allowed }_{k s} \\ 0, \text { otherwise }\end{array}\right.$

Where $\mathrm{k}$ allowed is the set of the remaining feasible states of the k-th exploratory ant. $\eta_{i s}(t)$ is the Local heuristic function of visibility, which is defined as:

$$
\eta_{i s}(t)=\frac{1}{d_{i j}}
$$

$\alpha, \beta$ represent the affecting weight of $\tau_{i j}(t)$ and $\eta_{i j}(t)$ on the transition probability, respectively. The pheromone update mechanism of elite ants is defined as

$\tau_{i j}(t+n)=(1-\rho) * \tau_{i j}(t)+\Delta \tau_{i j}(t)+\alpha \Delta \tau_{i j}^{i \epsilon \theta t}(t)$

Where $\rho(\rho \in(0,1))$ is the pheromone evaporation coefficient. In $\Delta \tau_{i j}(t)$ is pheromone increment of common 
ants which release on the path $<i, j>$ in the current iteration.? is the pheromone alpha factor of elite ant. $\Delta \tau_{i j}^{b e s t}(\mathrm{t})$ is pheromone increment of elite ants which release on the path $<\mathrm{i}, \mathrm{j}>$ in the current iteration.

$$
\Delta \tau_{i j}^{\text {best }}(t)=\left\{\begin{array}{c}
\frac{Q}{L_{b e s t}}, i_{x} j \text { fl }_{L_{t e s}} \\
0, \text { otherwise }
\end{array}\right\}
$$

Where $\mathrm{Q}$ is a constant, which represents the amount of the pheromone increment. $L$ best is the length of the path that the k-th lite ant has passed in the current iteration.

\section{B. Improved Ant Colony Algorithm}

We elaborate the improved ant colony optimization (IACO) algorithm for path planning as follows. It gives the distinction described in Fig. 2.

\section{Procedure IACO}

begin

Initialize parameters and pheromone trails

While (stopping criterion not satisfied) do

Position each ant in a starting node

Repeat

For each ant do

If (The ant is a common ant)

Chose next node by Eq. (1)

endif

if (The ant is an exploratory ant)

Chose next node by Eq. (5)

endif

endfor

until (Every ant has build a solution) if (The ant is superior than an elite ant) Replace the elite ant with the ant end if

Update the pheromone by Eq. (7) end while

end

\section{EXPERIMENT AND RESULTS}

We have developed three simulated grid examples to evaluate the IACO algorithm for grid scheduling. The experimental simulation is designed and implemented based on grid simulation architecture described in section 2.Experimental results are compared with the ACO algorithm and PFACO algorithm [15]. The initial parameters are set as follows: $\alpha=1, \rho=1, \rho=0.3, q=1$. The number of ants is 50 for ant colony algorithm and PFACO algorithm. The number of common ants and exploratory ants are 25 and 75, respectively. The number of iterations is 100 .

Table.1 parameters of PFACO algorithm

\begin{tabular}{|c|c|}
\hline Parameters & Value \\
\hline Gain coefficient of attractive force $k$ & 15 \\
\hline Initialization parameter $I$ & 0 \\
\hline Gain coefficient of repulsive force $m$ & 5 \\
\hline Obstacle distance $P o$ & 10 \\
\hline step length $L$ & 5 \\
\hline $\begin{array}{c}\text { The number of iterations of the artificial potential } \\
\text { field method } J\end{array}$ & 500 \\
\hline
\end{tabular}

In order to effectively test the performance of the IACO algorithm, multiple sets of test data are used (as shown in Fig.4). Experiments are tested for the optimal paths (as shown in Fig.5), convergence rates (as shown in Fig. 6), and the optimal path cost (as shown in tab. 2). 


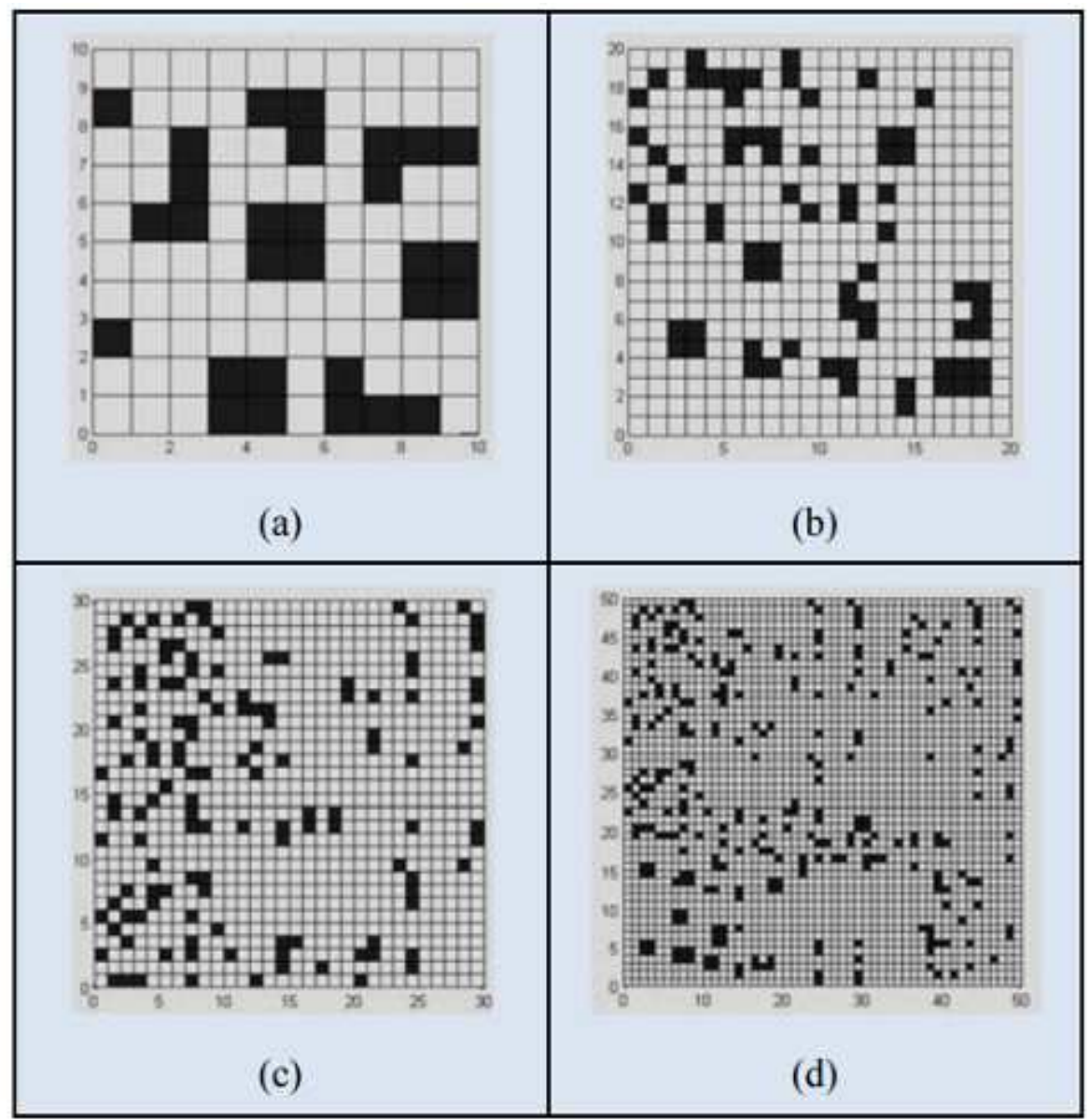

Fig. 4 Grid environment with obstacle

(a)10 110 grid (b) $20 \times 20$ grid. (c) $30 \times 30$ grid. (d) $50 \times 50$ grid

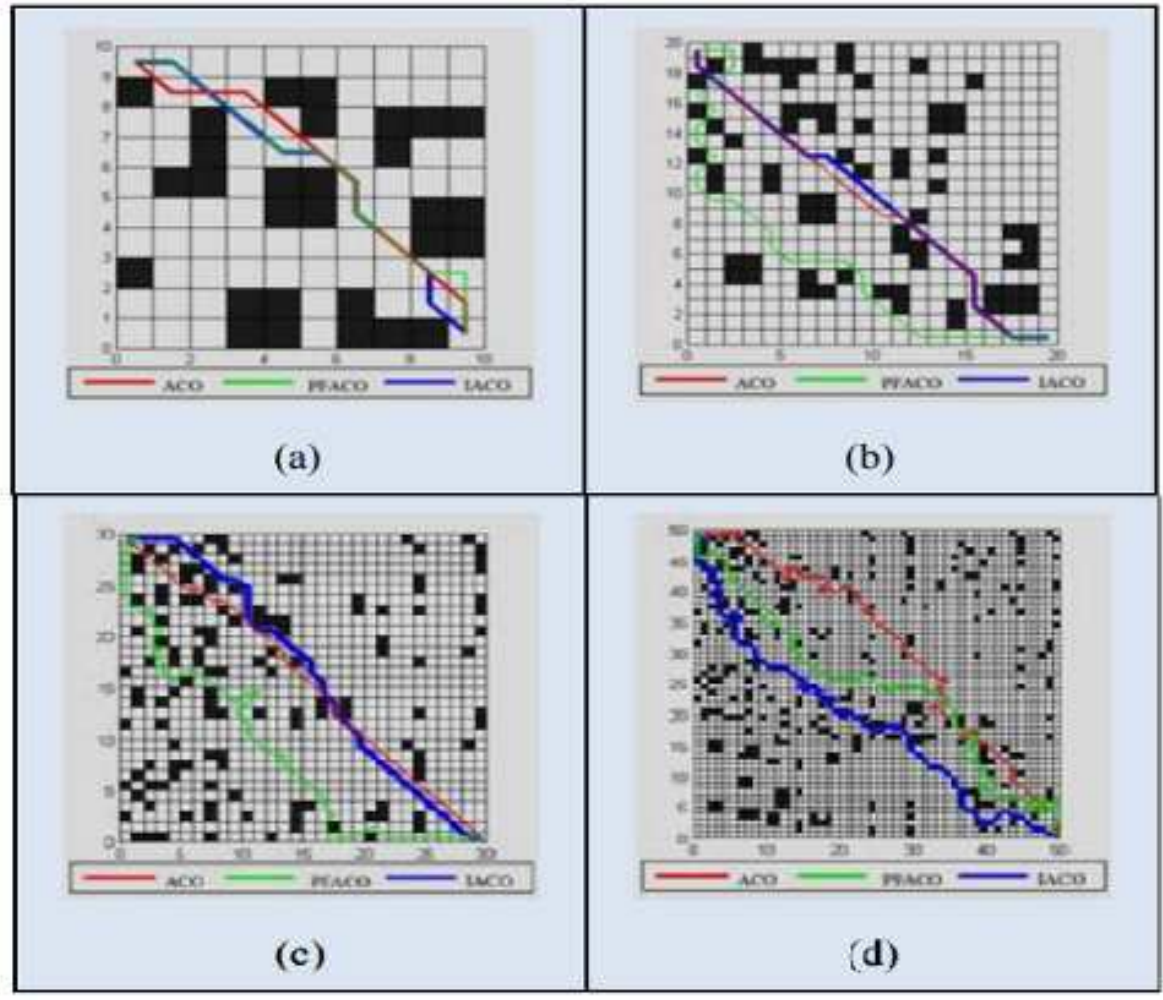

Fig.5. Optimal path

(a) Optimal path achieved by algorithms in $10 \times 10$ grid

(b) Optimal path achieved by algorithms in $10 \times 10$ grid

(c) Optimal path achieved by algorithms in $20 \times 20$ grid

(d) Optimal path achieved by algorithms in $20 \times 20$ grid. 


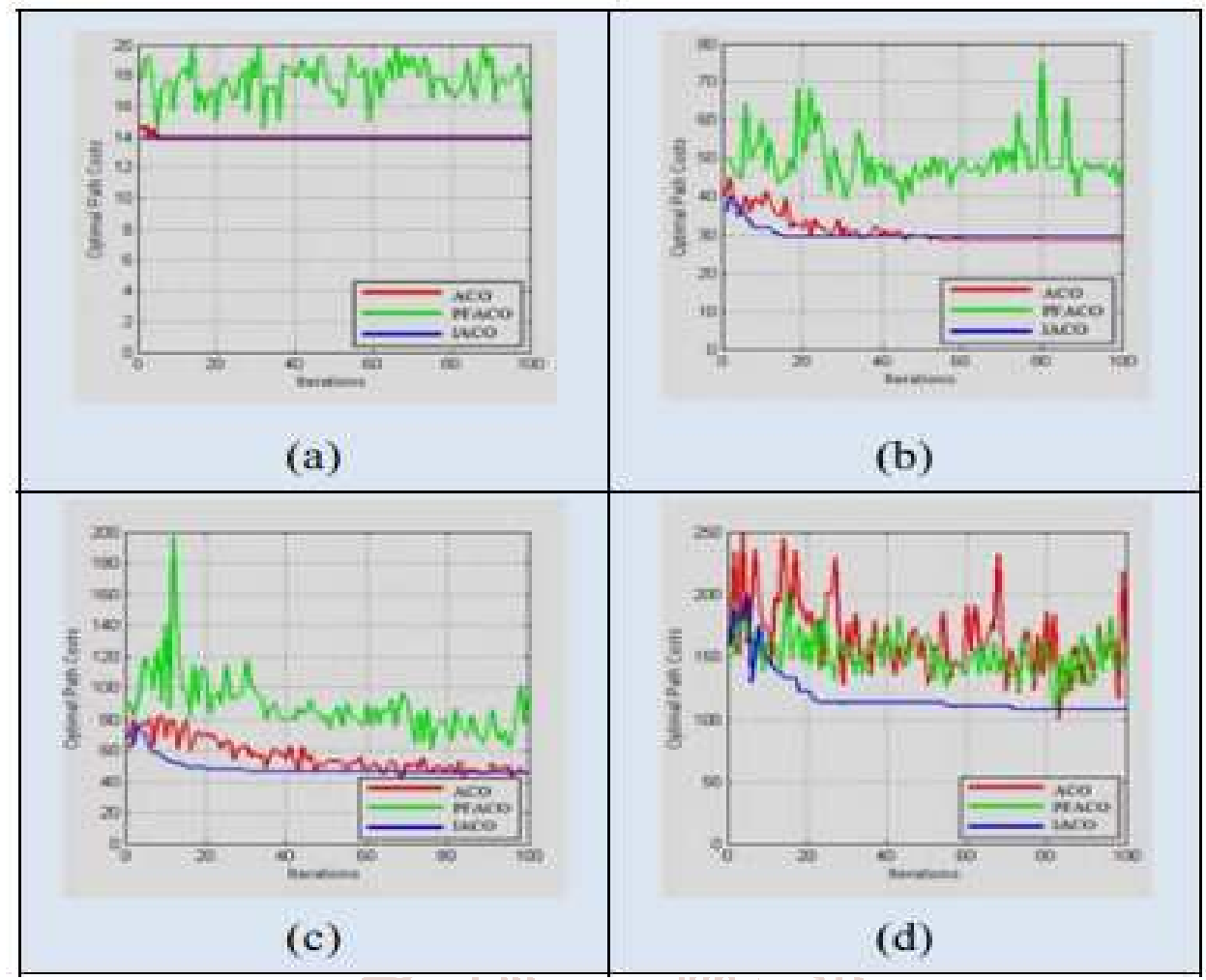

Fig. 6 Comparison of convergence curves

(a) Convergence curves achieved by algorithms in $10 \times 10$ grid

(b) Convergence curves achieved by algorithms in $20 \times 20$ grid

(c) Convergence curves achieved by algorithms in $30 \times 30$ grid

(d) Convergence curves achieved by algorithms in $50 \times 50$ grid

Experimental results show that the convergence speed of ant colony optimization algorithm is close to the convergence speed of improved ant colony optimization algorithm. With the expansion of the scale of the problem, the superiority of the IACO algorithm in the convergence speed is gradually reflected. he IACO algorithm can plan high quality feasible solutions in a short time. For $50 \times 50$ grid data, the ant colony cannot find feasible solutions within the maximum number of iterations. The stability of the PFACO is not so good. The results of simulation indicate that the improved ant colony algorithm is suitable for path planning in complex environment, which can find the feasible solution with higher convergence speed.

Table. 2 comparison of the improved ant colony algorithm and ant colony algorithm

\begin{tabular}{|c|c|c|c|}
\hline & $\begin{array}{c}\text { Optimal path cost } \\
\text { of } \\
\text { ACO algorithm }\end{array}$ & $\begin{array}{c}\text { Optimal } \\
\text { path cost of } \\
\text { PFACO } \\
\text { algorithm }\end{array}$ & $\begin{array}{c}\text { Optimal path cost } \\
\text { of } \\
\text { IACO algorithm }\end{array}$ \\
\hline $10^{*} 10$ & 13.899 & 13.899 & 13.899 \\
\hline $20 * 20$ & 28.627 & 38.627 & 28.627 \\
\hline $30^{*} 30$ & 46.527 & 59.355 & 42.184 \\
\hline $50 * 50$ & 96.125 & 109.761 & 94.326 \\
\hline
\end{tabular}

VI. CONCLUSION

While in a complex environment and the distance between the starting point and the target point is relatively long, ant colony algorithm is difficult to find a valid path and easy to fall into deadlock. An improved ant's colony optimization algorithm for path planning is presented in this paper. To avoid trapping into local optimum, a hybrid ant colony which is composed of common ants and exploratory ants is utilized in this paper. By improving the mechanism of updating pheromone and preserving the elite ants, the reasonable distribution of pheromone is realized, and the convergence rate of the algorithm is accelerated. Simulation results prove its effectiveness. Experimental results indicate that the algorithm is suitable for robot path planning in complex environment.

\section{REFERENCES}

[1] S. S. Ge, Y. J. Cui, "New potential functions for mobile robot path planning", IEEE Transactions on robotics and automation, Vol. 16,No. 5, pp. 615-620, 2000.

[2] S. K. Pradhan, D. R. Parhi, A. K. Panda, "Fuzzy logic techniques for navigation of several mobile robots", Applied soft computing, Vol. 9,No. 1, pp. 290-304, 2009.

[3] S. X. Yang, C. Luo, "A neural network approach to complete coverage path planning", IEEE Transactions on Systems, Man, and Cybernetics, Part B: Cybernetics, Vol. 34, No. 1, pp. 718-724, 2004.

[4] J. Tu, S. X. Yang, "Genetic algorithm based path planning for a mobile robot", IEEE International Conference on Robotics and Automation, pp. 1221-1226, 2003.

[5] M. Dorigo, V. Maniezzo, A. Colorni, "Ant system: optimization by a colony of cooperating agents", IEEE Transactions on Systems, Man, and Cybernetics, Part B: Cybernetics,, Vol. 26, No. 1, pp. 29-41,1996.

[6] P. Raja, S. Pugazhenthi, "Optimal path planning of mobile robots: A review", International Journal of Physical Sciences, Vol. 7, No. 9, pp.1314-1320, 2012. 
[7] M. P. Garcia, O. Montiel, O. Castillo, et al., "Path planning for autonomous mobile robot navigation with ant colony optimization and fuzzy cost function evaluation", Applied Soft Computing, Vol. 9,No. 3, pp. 1102-1110, 2009.

[8] X. Fan, X. Luo, S. Yi, et al., "Optimal path planning for mobile robots based on intensified ant colony optimization algorithm",International Conference on Robotics, Intelligent Systems and Signal Processing, pp. 131-136, 2003.

[9] K. K. Lim, Y.-S. Ong, M. H. Lim, et al., "Hybrid ant colony algorithms for path planning in sparse graphs", Soft Computing, Vol.12, No. 10, pp. 981-994, 2008.

[10] M. Brand, M. Masuda, N. Wehner, et al., "Ant colony optimization algorithm for robot path planning", International Conference on Computer Design and Applications (ICCDA), pp. 436-440, 2010.

[11] S. E. Muldoon, C. Luo, F. Shen, et al., "Naturally inspired optimization algorithms as applied to mobile robotic path planning",2014 IEEE Symposium on Swarm Intelligence (SIS), pp. 1-6, 2014.

[12] Q. Zhu, J. Hu, L. Henschen, "A new moving target interception algorithm for mobile robots based on subgoal forecasting and an improved scout ant algorithm", Applied Soft Computing, Vol. 13, No.1, pp. 539-549, 2013.

[13] R. Vatankhah, S. Etemadi, A. Alasty, et al., "Active leading through obstacles using ant-colony algorithm", Neurocomputing, Vol. 88, pp.67-77, 2012.

[14] J. Pan, X.-S. Wang, Y.-H. Cheng, "Improved ant colony algorithm for mobile robot path planning", Journal of China University of Mining \& Technology, Vol. 41, No. 1, pp. 108-113, 2012.

[15] J.-W. Lee, J.-J. Kim, B.-S. Choi, et al., "Improved ant colony optimization algorithm by potential field concept for optimal path planning", 8th IEEE-RAS International Conference on Humanoid Robots pp. 662667,2008 .

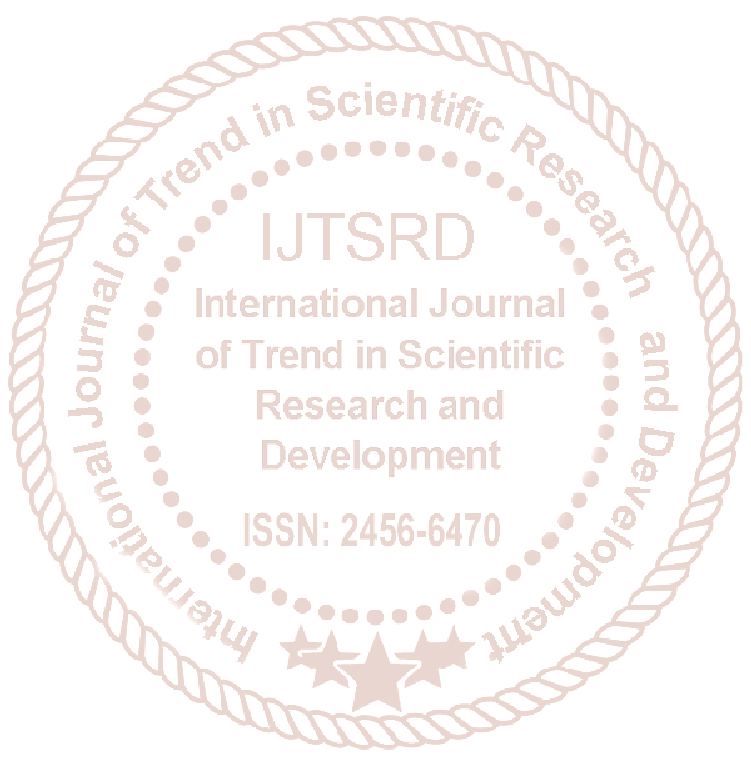

